\title{
Survival of Patients with Locally Advanced Triple Negative Breast Cancer After Neoadjuvant Platinum Containing Chemotherapy. Experience of a Single Institute
}

\author{
E. Ibrahim1, H. Ramadan', A. Arini' ${ }^{2}$ H. Mosleh $^{3}$ and N. AlNaimy ${ }^{4}$. \\ ${ }^{1}$ Oncology, ${ }^{2}$ Surgery, ${ }^{3}$ Pathology, ${ }^{4}$ Radiology, King Fahad Specialist Hospital, Dammam, Saudi Arabia
}

Background: Primary systemic chemotherapy is a standard approach to treating women with locally advanced breast cancers, with higher survival rates reported among patients who attain a pathological complete response. Triple negative breast cancer is a special biological entity that remains major challenge to oncologist. Around $12 \%-20 \%$ of breast cancers are triple negative. The current phase II study was conducted to evaluate the pathological complete response (pCR), efficacy and safety of anthracycline-taxane-cisplatinum containing regimen as neoadjuvant chemotherapy in locally advanced triple negative breast cancer.

Patients and Methods: This phase II study was conducted on eighteen women with stage III triple negative breast cancer who were recruited between July 2007 and February 2010 at King Fahad Specialist Hospital-Dammam, Saudi Arabia. Neoadjuvant chemotherapy consisted of 4 cycles of AC or FEC 100, followed by 4 cycles consisted of docetaxel-cisplatin $(75 \mathrm{mg} / \mathrm{m} 2$ each) every 3 weeks. Primary end point was pathological complete response. Kaplan-Meier method survival analysis was performed and group differences concerning survival time were evaluated by log-rank test.

Results: This is a preliminary report on the first eighteen pts included. Median age: 49 y 24-70); premenopausal: $16 ; 25 \%$ were below 35 years of age; Median tumor size: $9 \mathrm{~cm}$ ( 3.5-19); Grade III: 15; Stage IIIA: 3, IIIB:14, IIIC:1; all but 2 had positive nodes at diagnosis (89\%) . All pts completed the anthracycline part with 2 episodes of febrile neutropenia (F.N). Only 10 pts completed all 4 cycles of the second sequence with D-Cis; 4 completed 3 cycles. Toxicity related to D-Cis: F.N: 4; renal impairment: 2; Hypersensitivity reaction: 1 . No grade 5 toxicity. Clinical evaluation of response by RECIST criteria pre surgery: OR: 17/18 (94\%), CR: 9 (50\%); PR: 8 (44\%). The second sequence with D-Cis-T doubled the rate of clinical CR obtained with AC /FEC. One patient was not operated due to disease progression. Pathological assessment, revealed that $8(47 \%)$ pts had no residual invasive carcinoma in the breast; $3(18 \%)$ had residual occasional scattered tumor cells less than $5 \mathrm{~mm}$ (pT1a); 10 (59\%) had negative nodes; 8 achieved CpR and $2 \mathrm{nCpR}$. Patients with residual invasive component and /or nodal involvement had high baseline Ki 67 level. After a median follow up of 24 months, cumulative overall survival at 24 months is $88.9 \%$ for whole group. Cumulative overall survival in relation to response was $100 \%$ for patients who achieved pCR while overall cumulative survival rate for patients without $\mathrm{pCR}$ was $83.3 \%$ without statistical significance. Cumulative progression free survival was $100 \%$ for patients who achieved pCR while PFS was $65 \%$ in patients who did not achieve pCR without statistical significance.

Conclusion: This anthracycline-taxane-cisplatin based neoadjuvant chemotherapy regimen was well tolerated and achieved a high rate of $\mathrm{pCR} / \mathrm{npCR}$. Cumulative progression free survival was $100 \%$ for patients who achieved pCR while PFS was $65 \%$ in patients who did not achieve pCR without statistical significance.

Key words: Survival,Triple Negative,Breast Cancer,Cis Platinum

Corresponding Author: Ehab Ibrahim.

E-mail: ehabhas@hotmail.com

\section{INTRODUCTION}

Breast cancer is increasingly recognized as a heterogeneous disease exhibiting substantial differences with regard to biological behavior and requiring distinct therapeutic interventions ${ }^{1}$. Steroid hormone receptors, such as estrogen receptor (ER) and Progesterone Receptor (PR) and the oncogene ErbB-2/human epidermal growth factor receptor two (HER-2) are important factors in distinguishing breast cancer subtypes. In recent years, the term triple negative breast cancer has emerged to describe those cancers which do not express Estrogen Receptors (ER), Progesterone Receptors (PR) or overexpress human epidermal growth factor receptor $2(\mathrm{Her} 2)^{2}$. Triple negative breast cancer remains major challenge to oncologist and a source of great interest to laboratory investigators. A round $12 \%-20 \%$ of breast cancers are triple negative. Treatment options for triple negative breast cancers are 
limited because of lack of targeted treatment. Primary systemic chemotherapy is a standard approach to treating women with locally advanced breast cancers, with higher survival rates reported among patients who attain a pathological complete response. Pathological complete response $(\mathrm{pCR})$ provides a surrogate for disease-free and overall survival $^{3}$.

BRCA1 plays a central role in repair of doublestranded DNA breaks; a lack of BRCA1 therefore results in genomic instability thereby predisposing to the development of malignant disease. Triple negative breast cancer phenotype is particularly associated with BRCA1 mutations ${ }^{4}$. Similarly, about threequarters of BRCA1- related breast cancer exhibit a basal phenotype by gene expression microarray ${ }^{5}$ or immunohistochemistry $^{6}$, particularly among younger patients and patients with a family history of breast cancer who very often also present with p53 mutations. The association of TNBC with BRCA1 mutations and dysfunctional DNA repair may indicate an increased sensitivity toward DNA-damaging agents, i.e. platinum agents. A recent preclinical study demonstrated that overexpression of p63 (a p53-related transcription factor) and p73 (p53 associated as well) is common among TN cases and associated with sensitivity to cisplatin ${ }^{7}$. Clinical data regarding the use of platinum agents in TNBC are still limited.

The current phase II study was conducted to evaluate the pathological complete response (pCR), efficacy and safety of anthracycline-taxane-cisplatinum containing regimen as neoadjuvant chemotherapy in locally advanced triple negative breast cancer. All patients provided written consent.

\section{PATIENTS AND METHODS}

This phase II study was conducted on eighteen women with stage III triple negative breast cancer who were recruited between July 2007 and February 2010 at King Fahad Specialist Hospital-Dammam, Saudi Arabia. Neoadjuvant chemotherapy consisted of 4 cycles of AC or FEC 100, followed by 4 cycles consisted of docetaxel-cisplatin $\left(75 \mathrm{mg} / \mathrm{m}^{2}\right.$ each) every 3 weeks. Primary end point was pathological complete response. Secondary end points were safety and tolerability, complete and partial response rates, disease free survival, local and distant disease control.

\section{Eligibility criteria:}

Patients with stage III (A, B, C) locally advanced triple negative breast cancer histologically confirmed by surgical biopsy or core needle biopsy were included. Patients with age $>18$, Eastern Cooperation Oncology Group (ECOG) performance status $\leq 2$, life expectancy
$>12$ months, with adequate bone marrow reserve, adequate liver and renal function were eligible.

Exclusion criteria included prior chemotherapy or radiotherapy, clinically significant cardiac disease; severe renal or liver functions impairment and pregnant women.

Initial evaluation, assessment of clinical and pathological response.

Before entry, all patients underwent the followings: medical history, physical examination, tumor evaluation measurement and staging. Electrocardiogram (ECG), Left Ventricular Ejection Fraction (LVEF) evaluation by ECHO, complete blood cell count, serum chemistries, liver and renal function tests, bilateral mammography, computed topographic scan of chest, abdomen and pelvis. Complete blood cell count, renal functions and serum electrolytes were measured before the administration of each treatment cycle.

Clinical objective responses were evaluated according to Response Evaluation Criteria in Solid Tumor (RECIST) ${ }^{8}$. Complete Response (CR) was defined as the disappearance of all known disease with no evidence of progressive disease; Partial Response (PR) was defined as a $>30 \%$ decrease in the sum of longest diameters of all target lesions with no appearance of new lesions. Progressive Disease (PD) was defined as least $20 \%$ increase in the sum of the longest diameters of all target lesions or the appearance of new lesions. Stable disease was defined neither as PR nor as PD.

\section{Pathological assessment:}

Tumor samples were collected by needle core biopsy or surgical biopsy prior to systemic chemotherapy. ER, PR, HER-2/neu, Ki67 were determined by standard immunohistochemical methods. Immunohistochemical (IHC) analysis was performed using Rabbit monoclonal antibodies clones SPI and IE2 for ER and PR, respectively and (MIB-1) for ki67. Tumors with less than $1 \%$ stained cells were considered to have negative receptors status. HER-2/neu status was assessed by IHC using Rabbit monoclonal antibody clone 4B5 only if the results were 0 or $1+$ staining and by Fluorescence in Situ Hyperdization (FiSH) confirmation if $2+$ immunohistochemistry staining was present. In our study pathological response was considered complete ( $\mathrm{pCR}$ ) if no histological evidence of residual tumor or in situ tumor in all resected specimens of the breast and the axillary lymph nodes could be detected, near pathological response (npCR) was considered if only occasionally scattered invasive tumor cells with a size of $5 \mathrm{~mm}$ or less in aggregate in the tissue specimen removed from the breast at the time of definitive surgery. 


\section{Treatment plan and follow up:}

Neoadjuvant chemotherapy consisted of 4 cycles of AC $\left(60 \mathrm{mg} / \mathrm{m}^{2}, 600 \mathrm{mg} / \mathrm{m}^{2}\right)$ or $\mathrm{FEC}(500 \mathrm{mg} / \mathrm{m} 2,100 \mathrm{mg} /$ $\mathrm{m} 2,500 \mathrm{mg} / \mathrm{m} 2$ respectively) followed by 4 cycles consisted of docetaxel-cisplatin $\left(75 \mathrm{mg} / \mathrm{m}^{2}\right.$ each) every 3 weeks. Clinical response, complete blood count and chemistry were assessed with every cycle. Surgery was performed on day 28 after completion of preoperative chemotherapy. Adjuvant radiotherapy was administered after surgery. After completion of the treatment plan, follow up was every three months. Toxicity was assessed using the Common Toxicity Criteria Version two.

\section{RESULTS}

Eighteen women with stage III $(\mathrm{A}, \mathrm{B}, \mathrm{C})$ triple negative breast cancer were recruited between July 2007 and February 2010. Patient's clinical data are shown in (Table 1). Median age was 49 years with range of (24-70). Premenopausal were 16 patients; $25 \%$ of them below 35 years old, median tumor size: $9 \mathrm{~cm}$ $(3.5-19 \mathrm{~cm}) ; 15$ patients were grade III and all patients except two had positive lymph nodes. Tumor stage, histology, grade and ECOG P.S are shown in (Table 1). Clinical response rate was $88.8 \%(16 / 18)$ of which clinical complete response rate after anthracycline chemotherapy was $22.2 \%$ and reached up to $50 \%$ after

Table 1: patient's and tumor's characteristics.

\begin{tabular}{lc}
\hline Number of patients & 18 \\
\hline Menopausal status & 16 \\
\hline Pre & 2 \\
\hline Median age years(range) & $49(24-70)$ \\
\hline ECOG Performance status & 17 \\
\hline $0-1$ & 1 \\
\hline 2 & 3 \\
\hline Initial Stage TNM & 14 \\
\hline IIIA & 1 \\
\hline IIIB & \\
\hline IIIC & 18 \\
\hline Median tumor size (range) & 3 \\
\hline Histology & 15 \\
\hline Invasive duct carcinoma & 1 \\
\hline Grade & 15 \\
\hline II & 2 \\
\hline III & \\
\hline Type of surgery & \\
\hline Breast conservative surgery & \\
\hline Modified radical mastectomy & \\
\hline No operation & \\
\hline & \\
\hline
\end{tabular}

docetaxel-cisplatin chemotherapy. Progressive disease was detected in $11 \%$ of cases (two patients). All patients completed the anthracycline part with two episodes of febrile neutropenia. Only 10 patients completed all 4 cycles of Doc-Cisp; 4 patients completed 3 cycles. Toxicity related to Doc-Cisp was 4 cases with febrile neutropenia; two cases of reversible renal impairment and one case suffered from hypersensitivity reaction. Fifteen patients underwent modified radical mastectomy and one underwent breast conserving surgery. Pathological assessment revealed that $6(37.5 \%)$ patients had pathological complete response (no residual tumor cell in the breast or the axillary lymph nodes); 3 (18\%) had residual scattered tumor cells less than $5 \mathrm{~mm}$ (near pathological complete response); 8/16 (50\%) had negative lymph nodes. Patients with residual invasive component and/ or nodal involvement had high baseline Ki67 level. Median follow up consisted of 24 months with range of (8-40). Cumulative overall survival at 24 months was $88.9 \%$ for the whole group (Fig. 1). OS rate in relation to response was $100 \%$ for patients who achieved pCR while OS rate for patients without $\mathrm{pCR}$ was $83.3 \%$ without statistical significance ( $\mathrm{p}$ value 0.15) (Fig. 2). Cumulative progression free survival was $100 \%$ for patients who achieved pCR while PFS was $65 \%$ in patients who did not achieve pCR without statistical significance (P value 0.12) (Fig. 3).

Table 2: Clinical and pathologic response after neoadjuvant chemotherapy.

\begin{tabular}{lc}
\hline Response & No. of Pts (\%) \\
\hline Clinical response after ACor FEC & $16 / 18(88.8 \%)$ \\
\hline Complete response & $4(22.2 \%)$ \\
\hline Partial response & $12(66.6 \%)$ \\
\hline Clinical response after cisplatin-docetaxel & $16 / 18(88.8 \%)$ \\
\hline Complete response & $9(50 \%)$ \\
\hline Partial response & $7(38.8 \%)$ \\
\hline Progressive disease & $2(11.1 \%)$ \\
\hline Pathological response & $8 / 16(50 \%)$ \\
\hline Complete pathological response (cPR) & $6 / 16(37.5 \%)$ \\
\hline Near complete pathological response (ncPR) & $3 / 16(18.7 \%)$ \\
\hline Negative axillary lymph nodes & \\
\hline
\end{tabular}


Overall Survival

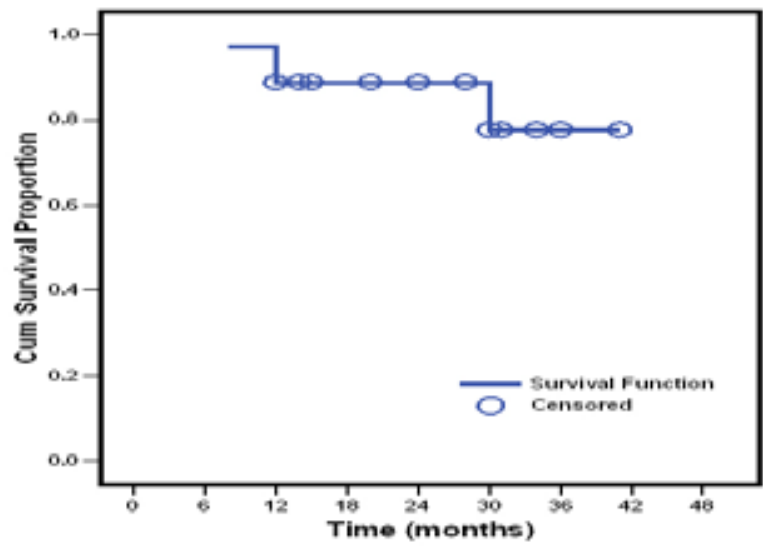

Figure 1: Overall survival for whole group.

Overall Survival

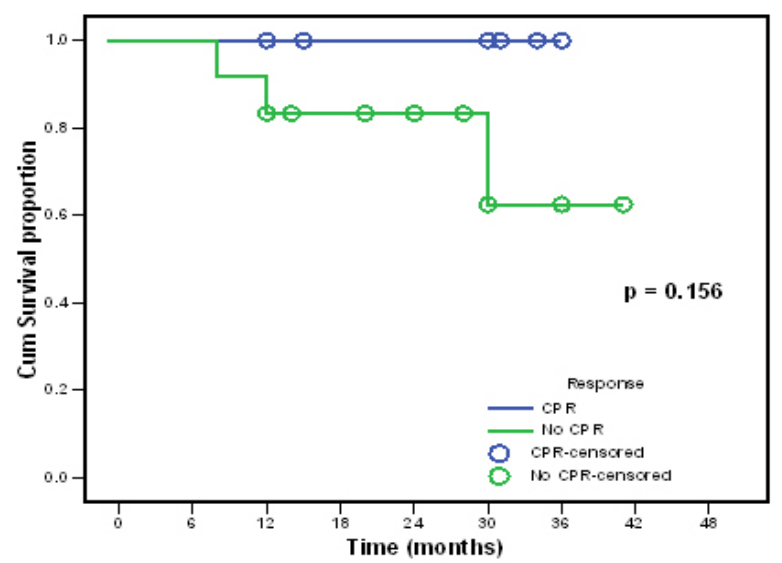

Figure 2: cumulative overall survival in relation to pathological response.

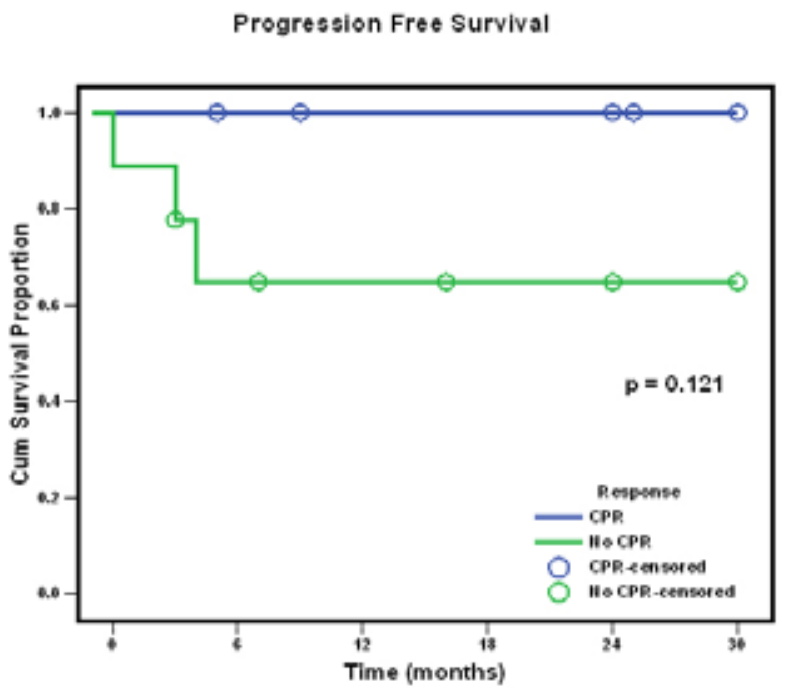

Figure 3: cumulative progression free survival in relation to pathological response.

\section{DISCUSSION}

Triple negative breast cancer (TNBC) is an aggressive subtype of breast cancer that lacks a therapeutic target, making chemotherapy the primary systemic modality used in the treatment of this disease. The use of neoadjuvant chemotherapy for this sub-group of patients has risen in recent years, likely as a result of recent studies demonstrating that triple negative breast cancer is more chemosensitive than other subtypes of breast cancer ${ }^{9}$. Further, the response of the tumor to neoadjuvant chemotherapy provides important prognostic information, whereby patients with a pCR in both the breast and axillary lymph nodes fare better than those with residual disease ${ }^{10}$. TNBC is a high risk breast cancer in view of younger age, poorly differentiated tumors and shortened survival. Bauer et al., study showed that patients with triple negative phenotype were significantly more likely to be under age 40 , had more advanced stage at diagnosis, were more likely to have a poorly differentiated histology and regardless of stage at diagnosis, women with triple negative breast cancer had poorer survival than those with other breast cancer ${ }^{11}$. In our study $25 \%$ of our patient below 35 years, $83 \%$ were grade 3 , median tumor size was $9 \mathrm{~cm}$ and $77 \%$ of patients had stage IIIB. Given the importance of chemotherapy in this disease, investigators have focused on optimizing drug selection of existing chemotherapeutic agents. From a biological standpoint, DNA damaging agents such as platinum agents are of high priority based upon the BRCA1 pathway and DNA repair dysfunction in this subtype as described above, which may enhanced sensitivity to DNA- damaging agents ${ }^{12}$. It has been suggested that younger women receive greater benefits from treatment with neoadjuvant therapy than older

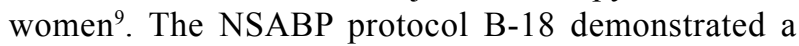
trend in favor of preoperative chemotherapy for DFS and $\mathrm{OS}$ in women less than 50 years old ${ }^{13}$. It is clear from previous studies that TNBCS display higher rates of $\mathrm{pCR}$ following neoadjuvant chemotherapy, a major determinant of clinical outcome, than non$\mathrm{TNBCS}^{14}$. However, in the setting of residual disease after neoadjuvant treatment, the overall prognosis of this disease is still poor. Carey et al., found that TNBC and HER2+ breast cancers display much higher rates of pCR $(27 \% \text { and } 36 \% \text {, respectively })^{15}$. Additionally, Liedtke et al., performed large study of 1118 patients with early-stage breast cancer and found that patients with TNBC had significantly higher pCR rates ${ }^{10}$. The clinical activity of cisplatin and carboplatin against unselected breast cancer was found many years ago to be modest with an overall response rate of $32 \%-54 \%{ }^{16}$. Garber neoadjuvant trial single agent cisplatin in 28 women with triple 


Vol. 7 | No. 3-4 $2011 \quad$ Ehab Ibrahim, et al.

negative breast cancer demonstrated $22 \% \mathrm{pCR}^{17}$ and another neoadjuvant trial in triple negative disease in which 9 of 10 had pCR to single agent cisplatin ${ }^{18}$. Taxanes and anthracycline are active in TNBC and remain important agents. The addition of platinum analogue to other chemotherapeutic agents appears to benefit patients with or without triple negative breast cancer. In our study the clinical complete response rate after anthracycline was $22 \%$ and doubled after administration of cisplatinum -taxane reached to $50 \%$. Pathological complete response rate is $37.5 \%$ and if we added cases with npCR the pathological response rate reached to $56 \%$. $50 \%$ of excised axillary lymph nodes are negative for malignant cells. Sirohi et al., reported in his study that overall response rate (CR,PR) was $100 \%$ in triple negative tumors and pathological complete response was $17 \%$ while 5 -years overall survival and DFS rates were $65 \%$ and $57 \%{ }^{19}$. We reported in our study that 2- years overall survival for whole group was $88.9 \%$ while overall survival regarding pCR was $100 \%$ and $83.3 \%$ in group without pCR without statistical significant. Also regarding PFS was $100 \%$ for group with pCR while PFS was $65 \%$ for group without pCR. Our results suggest that there may indeed be some clinical gain with platinum salt chemotherapy for triple negative tumors also tumor response is an important surrogate for patient's survival. This anthracycline-taxane-cisplatin based neoadjuvant chemotherapy regimen was well tolerated and achieved a high $\mathrm{pCR}$ rate.

\section{REFERENCES}

1. Glass AG, Lacey Jr. JV, Carreon JD, Hoover RN. Breast cancer incidence, 1980-2006: Combined roles of menopausal hormone therapy, screening mammography and estrogen receptor status. J.Natl.Cancer Inst. 2007;99(15):1152-61

2. Effects of chemotherapy and hormonal therapy for early breast cancer on recurrence and 15-year survival: An overview of the randomised trials. Lancet 2005;365(9472):1687-717.

3. Nanda R, Olopade F. Intro to December focus series on breast cancer. Clin.Cancer Res. 2008:14.

4. Lakhani SR, Van De Vijver MJ, Jacquemier J, Anderson TJ, Osin PP, McGuffog L, et al. The pathology of familial breast cancer: Predictive value of immunohistochemical markers estrogen receptor, progesterone receptor, HER-2 and p53 in patients with mutations in BRCA1 and BRCA2. J.Clin.Oncol. 2002;20(9):2310-8.

5. Sørlie T, Perou CM, Tibshirani R, Aas T, Geisler S, Johnsen $\mathrm{H}$, et al. Gene expression patterns of breast carcinomas distinguish tumor subclasses with clinical implications. Proc.Natl.Acad.Sci.U.S.A. 2001;98(19):10869-74.

6. Foulkes WD, Brunet JS, Stefansson IM, Straume O,
Chappuis PO, Bégin LR, et al. The prognostic implication of the basal-like (cyclin E high/p27low/p53 +/glomeruloidmicrovascular-proliferation+) phenotype of BRCA1related breast cancer. Cancer Res. 2004;64(3):830-5.

7. Leong CO, Vidnovic N, DeYoung MP, Sgroi D, Ellisen LW. The p63/p73 network mediates chemosensitivity to cisplatin in a biologically defined subset of primary breast cancers. J.Clin.Invest. 2007;117(5):1370-80.

8. Therasse P, Arbuck SG, Eisenhauer EA, Wanders J, Kaplan RS, Rubinstein L, et al. New guidelines to evaluate the response to treatment in solid tumors. J.Natl.Cancer Inst. 2000;92(3):205-16.

9. Rastogi P, Anderson SJ, Bear HD, Geyer CE, Kahlenberg MS, Robidoux A, et al. Preoperative chemotherapy: Updates of national surgical adjuvant breast and bowel project protocols B-18 and B-27. J.Clin.Oncol. 2008;26(5):778-85.

10. Liedtke C, Mazouni C, Hess KR, André F, Tordai A, Mejia $\mathrm{JA}$, et al. Response to neoadjuvant therapy and long-term survival in patients with triple-negative breast cancer. J.Clin.Oncol. 2008;26(8):1275-81.

11. Bauer KR, Brown M, Cress RD, Parise CA, Caggiano V. Descriptive analysis of Estrogen Receptor (ER)-negative, Progesterone Receptor (PR)-negative and HER2-negative invasive breast cancer, the so-called triple-negative phenotype: A population-based study from the California Cancer Registry. Cancer 2007;109(9):1721-8.

12. Rouzier R, Perou CM, Symmans WF, Ibrahim N, Cristofanilli $\mathrm{M}$, Anderson $\mathrm{K}$, et al. Breast cancer molecular subtypes respond differently to preoperative chemotherapy. Clin.Cancer Res. 2005;11(16):5678-85.

13. Wolmark N, Wang J, Mamounas E, Bryant J, Fisher B. Preoperative chemotherapy in patients with operable breast cancer: Nine-year results from National Surgical Adjuvant Breast and Bowel Project B-18. J.Natl.Cancer Inst.Monogr. 2001(30):96-102.

14. Ferrière JP, Assier I, Curé H, Charrier S, Kwiatkowski F, Achard JL, et al. Primary chemotherapy in breast cancer: Correlation between tumor response and patient outcome. Am.J.Clin.Oncol.Cancer Clin.Trials 1998;21(2):117-20.

15. Carey LA, Dees EC, Sawyer L, Gatti L, Moore DT, Collichio F, et al. The triple negative paradox: Primary tumor chemosensitivity of breast cancer subtypes. Clin. Cancer Res. 2007;13(8):2329-34.

16. Smith IE, Walsh G, Jones A, Prendiville J, Johnston S, Gusterson B, et al. High complete remission rates with primary neoadjuvant infusional chemotherapy for large early breast cancer. J.Clin.Oncol. 1995 Feb;13(2):424-9.

17. Garber JE, Richardson A, Harris LN, et al. Neoadjuvant cisplatin (CDDP) in triple-negative Breast Cancer (BC). 29th Annual San Antonio Breast Cancer Symposium; 2006.

18. Byrski T, Gronwald J, Huzarski T, Grzybowska E, Budryk M, Stawicka M, et al. Response to neo-adjuvant chemotherapy in women with BRCA1-positive breast cancers. Breast Cancer Res.Treat. 2008;108(2):289-96.

19. Sirohi B, Arnedos M, Popat S, Ashley S, Nerurkar A, Walsh G, et al. Platinum-based chemotherapy in triplenegative breast cancer. Ann.Oncol. 2008;19(11):1847-52. 